\title{
Investigation of stationary-crossflow-instability induced transition with the temperature-sensitive paint method*
}

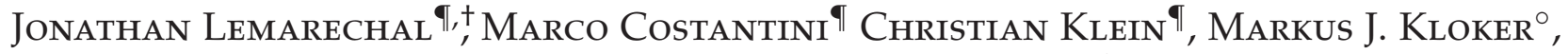 \\ Werner Würz ${ }^{\circ}$, Holger B.E. Kurz ${ }^{\circ}$, Thomas Streit ${ }^{\S}$, Sven Schaber ${ }^{*}$ \\ I Deutsches Zentrum für Luft- und Raumfahrt e.V. (DLR), \\ Bunsenstraße 10, D-37073 Göttingen, Germany, jonathan.lemarechal@dlr.de \\ ${ }^{\circ}$ Institut für Aerodynamik und Gasdynamik, Universität Stuttgart \\ Pfaffenwaldring 21, D-70569 Stuttgart, Germany \\ § Deutsches Zentrum für Luft- und Raumfahrt e.V. (DLR), \\ Lilienthalplatz 7, D-38108 Braunschweig, Germany \\ *Airbus Operations, \\ Airbus-Allee 1, Bremen, Germany,
}

\begin{abstract}
The Temperature-Sensitive Paint (TSP) method is used for surface-based flow visualizations on a swept-wing wind-tunnel model with a generic natural laminar-flow airfoil. Within the investigated parameter range the stationary crossflow instability is the dominating instability mechanism. Based on the TSP results the location of the laminar-turbulent transition and the most amplified wavenumber of the stationary crossflow instability are determined. The test is performed with three different conditions of the leading-edge surface: highly polished, unpolished, and highly polished with discrete roughness elements applied. The Temperature-Sensitive Paint method has proven to have sufficient spatial resolution and temperature sensitivity to resolve skin friction variations to detect the footprint of stationary crossflow vortices even inside of turbulent wedges. With the discrete roughness elements, i.e. cylindrical elements with micron-sized height, the transition could be delayed successfully for certain conditions. Local low-frequency movement of the beginning of turbulent wedges was detected for some data points with an unpolished leading edge.

Keywords - laminar-turbulent transition, stationary crossflow instability, upstream flow deformation, UFD, discrete roughness elements, DRE, transition delay, temperature-sensitive paint method, TSP, swept-wing wind-tunnel model de$\operatorname{sign}$
\end{abstract}

\section{INTRODUCTION}

A swept wing inherently develops a threedimensional boundary layer, which is prone to crossflow (CF) instability, Tollmien-Schlichting instability, centrifugal instability and attachment line instability Saric et al. (2011). The crossflow instability is of great interest for the development of laminar wings either by natural laminar flow (NLF) or hybrid laminar flow control (HLFC) because it leads to a very early laminar-turbulent transition of the boundary layer on a swept wing. For swept

\footnotetext{
*Citation and credit: Lemarechal, J., Costantini, M., Klein, C., Kloker, M.J., Würz, W., Kurz, H.B.E., Streit, T., Schaber, S.: Investigation of stationary-crossflow-instability induced transition with the temperature-sensitive paint method. Experimental Thermal and Fluid Science 109 (2019) 109848 doi:10.1016/j.expthermflusci.2019.109848

${ }^{+}$Corresponding author
}

wings with a sweep angle $\varphi>15^{\circ}$ the crossflow instability is limiting the extent of laminar flow for large Reynolds numbers Schrauf (2005). Important receptivity mechanisms with respect to the crossflow instability are driven by surface roughness and the turbulence intensity. The turbulence level governs the mode selection, i.e., dominating traveling or stationary crossflow modes, and also changes the initial amplitude caused by surface roughness Hunt and Saric (2011). Alongside the stationary-mode instability also a traveling-mode instability exists, being dominant in flows with increased turbulence intensity Devhle and Bippes (1996). The receptivity to surface roughness is the most important mechanism for feeding the stationary CF instability in a low turbulence environment, i.e. even smallest surface roughness causes significant initial amplitudes of CF modes to develop in a three-dimensional boundary 
layer Müller and Bippes (1989). Surface roughness has an especially large effect on the stationary crossflow instability close to the indifference point (critical Reynolds number) Radetzsky et al. (1999). Measurements performed in flight reported the most amplified stationary-crossflow wavelengths in the order of $\lambda \approx 2 \mathrm{~mm}-5 \mathrm{~mm}$ Schrauf et al. (1998); Herbert and Schrauf (1996). Further details on crossflow instabilitv are given bv Bippes (1999); Saric et al. (2003), and on crossflow receptivity by Kurz and Kloker (2014).

The stationary CF instability is characterized by standing co-rotating vortices inside the boundary layer. These vortices redistribute high- and lowmomentum fluid and thus strongly deform the mean velocity profile. Initially, the stationary crossflow vortices grow exponentially, until at some point their growth saturates. This change to nonlinear growth happens far upstream of the transition location. Finally, the transition is initialized by a high-frequency secondary instability developing inside the crossflow vortices. It is located in the updraft part of the stationary crossflow vortices in the mid height of the boundary layer Kohama et al. (1991). In experiments the high-frequency secondary instability are predominantly the ' $z$ ' modes Wassermann and Kloker (2002), which are typically in the $\mathrm{kHz}$ regime, grow rapidly and cause local turbulence. Because of the local development of secondary instability inside the stationary crossflow vortices the transition front is spatially nonuniform in spanwise direction and a sawtooth pattern develops.

The receptivity to surface roughness can be used to delay laminar to turbulent transition by applying discrete roughness elements (DRE) Saric et al. (2011); Radetzsky et al. (1999). Appropriate DRE trigger a higher-wavenumber mode, which grows initially stronger than the natural mode, that typically grows strongest on the long run. This delays the transition by nonlinear suppression of the natural mode. The method has been termed also Upstream Flow Deformation (UFD) by Wassermann and Kloker (2002).

Previous experiments showed that it is challenging to delay laminar-turbulent transition by applying DRE in the low-disturbance environment that aircraft encounter in flight Saric et al. (2015). The DRE height is only in the order of a few microns Radetzsky et al. (1999). The initial amplitude generated by the DRE decreases with decreasing turbulence level Hunt and Saric (2011), which reduces the effectiveness of DRE to delav the laminar-turbulent transition Hunt and Saric (2011); Woodruff et al. (2011).
Measurement techniques usually used to investigate

crossflow-induced transition are on one hand direct quantitative methods such as hot-wire Müller and Bippes (1989) and hot-film anemometrv NitschkeKowsky and Bippes (1988). On the other hand more qualitative surface visualizations are used like infrared-thermography, which is used in wind tunnels Ouast (1987); Petzold and Radespiel (2015) as well as in flight Crawford et al. (2013), or sublimating chemicals Müller and Bippes (1989); Kohama et al. (1991).

The crossflow instability is especially difficult to investigate in wind tunnels. The demand for a very smooth surface causes high requirements on the model manufacturing and the measurement techniques used to investigate the crossflow instability. Besides, the transfer of the results from wind tunnel experiments to flight conditions is difficult because of differing turbulence intensity.

The flow visualization techniques provide limited information compared to velocity measurements but the flow is characterized synchronously and continuously for a large area. The thermographic measurement techniques typically require an artificial temperature difference between model surface and flow for the visualization Fey and Egami (2007). The artificial temperature difference generates a heat flux which results in different surface temperature in regions with high and low skin friction. This allows to distinguish between laminar and turbulent flow. A surface with good thermal resistance and low reflection of infrared radiation is necessary, which is usually achieved by paint or foil added to the surface of the model.

Visualizations with the sublimating chemical methods also visualize differences in skin friction with a chemical, e.g. Naphthalene Kohama et al. (1991), that evaporates more quickly in regions of larger wall shear stress. A homogeneous thickness, which is reported in the order of 5 to $13 \mu \mathrm{m}$ Downs III (2012); Holmes et al. (1984), of the chemical is necessary. This layer thickness is equal to the roughness identified as already sensitive for the crossflow instability by Radetzsky et al. (1999). Therefore, the first $25 \%$ of the airfoil remains uncoated Downs III (2012) but this region is of importance due to the receptivity to surface roughness in this region and primary growth of the vortices.

Another visualization technique is the Temperature-Sensitive Paint (TSP) method which is also a non-intrusive thermographic measurement technique. It provides high spatial resolution over 
large areas. The TSP method can be used to measure the surface temperature distribution of a wind tunnel model due to a temperature-dependent fluorescence of molecules called luminophores. The luminophores are excited by light of an appropriate wavelength and the emitted light, which has a larger wavelength than the excitation light, is recorded. Typically, a light emitting diode (LED) is used to provide the excitation light and a charge-coupled device (CCD) or complementary metalâĂŞoxideâĂŞsemiconductor (CMOS) camera is used for recording. The TSP technique is an established measurement technique for a variety of applications, e.g. in cryogenic facilities (Fev et al., 2007), rotating svstems (Weiss et al., 2017), and in water facilities (Fey et al., 2013). Details about the photophysics of TSP and more information on TSP measurements are given by Liu and Sullivan (2005) and further details on the application of the TSP method for the visualization of laminar-turbulent transition in low-speed tests are given by Fey and Egami (2007).

In this paper the capabilities of the TSP method to investigate stationary $\mathrm{CF}$ instability modes are presented. The laminar-turbulent transition, the CF vortices' footprints, and the influence of the model heating on the measured results are experimentally studied in this work. Furthermore, time-resolved TSP measurements were performed to investigate an unsteady behavior of the onset of laminar-turbulent transition. In the present experiment three different conditions of the leading-edge surface were examined: highly polished, highly polished with spraycoated DRE, and unpolished. The DRE were applied to study the transition delay capabilities.

\section{EXPERIMENTAL SETUP AND DATA ACQUISITION}

\subsection{Laminar Wind Tunnel}

The presented experiment was conducted in the Laminar Wind Tunnel (LWT) of the Institute of Aerodynamics and Gas Dynamics, University of Stuttgart. The LWT is of Eiffel tvpe with an open-return Wortmann and Althaus (1964). The closed test section is $0.73 \mathrm{~m}$ high, $2.73 \mathrm{~m}$ wide, and $3.15 \mathrm{~m}$ long. The span of the vertically mounted airfoils is $0.73 \mathrm{~m}$ and posed a challenge for designing a wind tunnel model with the characteristics of an infinite swept wing flow. The LWT has a very good flow quality with a turbulence level of $\mathrm{Tu}=0.02 \%$ at $30 \mathrm{~m} / \mathrm{s}$ Herr (2004) and is thus well suited for the investigation of stationary CF insta-

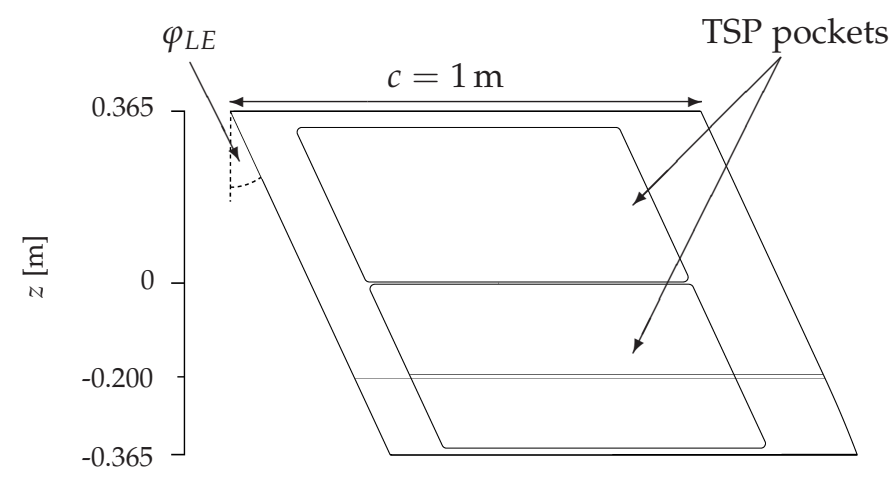

Figure 1: Side view of the pressure side of the wind tunnel model.

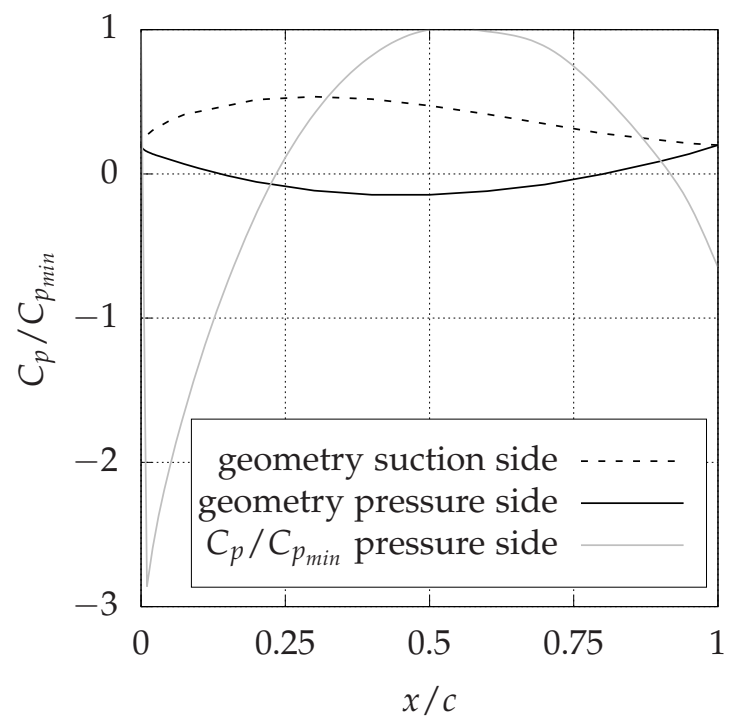

Figure 2: Model cross section and measured normalized pressure distribution $C_{p} / C_{p_{\min }}$ on the pressure side at $\alpha=6^{\circ}$ and $\operatorname{Re}_{\mathrm{C}}=4.61 \cdot 10^{6}$.

bilities. Most energy of the turbulent fluctuations is contained in low frequency fluctuations in the range of $f=10 \mathrm{~Hz}-150 \mathrm{~Hz}$ Plogmann et al. (2015).

\subsection{Wind tunnel model}

For this test a generic swept-wing airfoil with instability characteristics similar to free flight was designed Schrauf and Furtado Ferreira (2011). In this work this initial airfoil geometry was adapted especially to the constraints of the LWT's test section in a numerical design process to maintain the amplification factor (N-factor) distribution and avoid strong suction peaks.

A swept wing with a sweep angle of $\varphi_{L E}=25^{\circ}$, a 
span of $0.73 \mathrm{~m}$ and a chord length of $c=1 \mathrm{~m}$, was selected, see figure 1 The large chord length allows obtaining Reynolds numbers up to $\operatorname{Re}_{c}=5 \cdot 10^{6}$, however the small aspect ratio implies a large influence of the wind tunnel walls. The target of the design was to obtain a large spanwise region of the wing on the pressure side with similar $\mathrm{N}$-factors as the ones provided by the infinite swept wing solution of the corresponding airfoil. Furthermore, the suction side of the wind tunnel model had to be designed without flow separation in the relevant parameter range. The former can be achieved by a geometry which generates a spanwise-constant pressure distribution. Despite the three-dimensional influence of the narrow test section the following approaches can be taken: a) swept wing with spanwise constant airfoil sections and adaptive walls Birkemever (1991), or b) using 3D inverse designs, in this case the swept wing has spanwise changing sectional airfoils Streit and Hoffrogge (2017). In this work the design task was to be achieved with a simple geometry and therefore, the airfoil shown in figure 2 was designed which is used in the span region $-0.2 \mathrm{~m}<z<0.365 \mathrm{~m}$. The cross section is shown in figure 2. It provides a favorable pressure gradient on the pressure side, which is the side under investigation in this work, up to $x / c \approx 0.5$ at $\alpha=6^{\circ}$. This enables strong growth of stationary $\mathrm{CF}$ instability as shown in the stability diagram in figure 3 , and surpresses Tollmien-Schlichting instability. The neutral point for $\mathrm{CF}$ instability is located at $x / c=0.02$ in this case. Please note that positive angles of attack are investigated; therefore, the lower side of the model is the pressure side.

A second airfoil was designed for the region close to the bottom wind tunnel wall, where the intersection of wind tunnel model and wall is like a forward swept-wing at $z=-0.365 \mathrm{~m}$. Here the initial geometry showed a too large suction peak. In the region $-0.2 \mathrm{~m} \geq z \geq-0.365 \mathrm{~m}$ the section airfoil geometry is blended between the two airfoils. Additional twist was introduced, which changes linearly from $7^{\circ}$ at $z=-0.365 \mathrm{~m}$ to $0^{\circ}$ at $z=-0.2 \mathrm{~m}$, to further reduce the leading edge suction peak. Since different surface conditions were tested in the experiment, the airfoil was twisted around the nose, in order to keep the leading edge geometry constant.

In contrast to an infinite swept wing, a swept wing in a wind tunnel does not have an effective sweep $\varphi_{\text {eff }}$ which coincides with the geometrical sweep angle Redecker and Wichmann (1991). This is an important aspect since the CF-instability is very sensitive to the effective local sweep angle. Figure 4 shows the

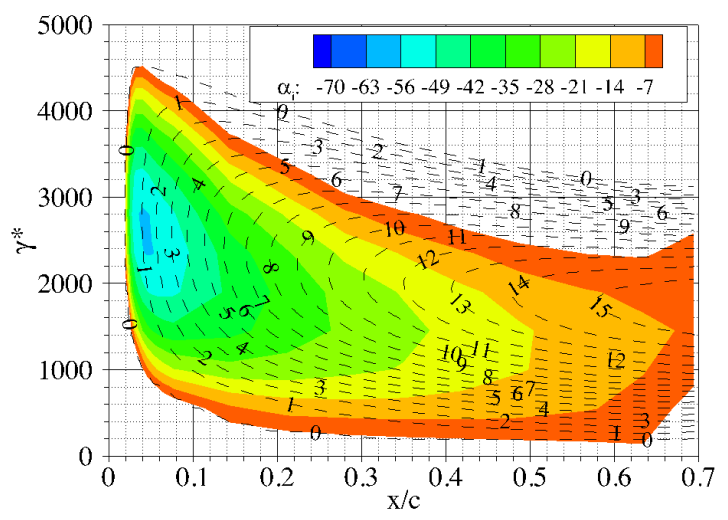

Figure 3: For $R e_{c}=5 \cdot 10^{6}$ and an angle of attack $\alpha=6^{\circ}$ the stability diagram shows the stationary crossflow modes $(\omega=0)$, the spatial amplification rate $\alpha_{i}$ (colored contours), and the $n$-factor (dashed lines).

effective sweep angle as a function of the spanwise position $z$ in comparison to the leading edge sweep angle $\varphi_{L E}$. The sweep angle was obtained from local attachment stagnation line pressure. Note that there is a strong influence of the wind tunnel walls on the effective sweep, especially in regions: $0.365 \mathrm{~m} \leq z \leq$ $0.2 \mathrm{~m}$ and $-0.2 \mathrm{~m} \leq z \leq-0.365 \mathrm{~m}$. In the mid span region $|z| \leq 0.2 \mathrm{~m}$ the differences between the effective sweep angle and the infinite sweep angle of $\varphi_{L E}=$ $25^{\circ}$ are smaller as can be seen in figure 4

For $\operatorname{Re}_{c}=5 \cdot 10^{6}$ and an angle of attack of $\alpha=6^{\circ}$ a most amplified dimensionless stationarycrossflow wavenumber of $\gamma^{*}=2 \pi /\left(\lambda^{*} c \cos \varphi_{L E}\right) \approx$ 1900 was achieved, which is in good agreement with the wavenumbers reported at free flight conditions. Based on the stability diagramm (figure 3) one can identify a possible mode range for application of the UFD method. Especially wavenumbers between $2400 \leq \gamma^{*} \leq 2900$ are initially growing the most and are therefore good candidates as UFD modes, see figure 3. Further downstream these modes are damped and do not reach the critical $\mathrm{N}$-factors, i.e. they are not expected to lead to laminar-turbulent transition.

The wind tunnel model is composed of two aluminum shells bolted together while the inside is hollow. A thin wall thickness of the model was chosen to improve model handling, which required additional stiffeners, i.e., spars and ribs. The TSP coating was embedded in two pockets (see figure 1), which were machined into the model surface, in order to maintain the designed contour and avoid surface imperfections. In chordwise direction, the TSP pockets range from $x / c=0.12$ to $x / c=0.81$ and in spanwise direc- 


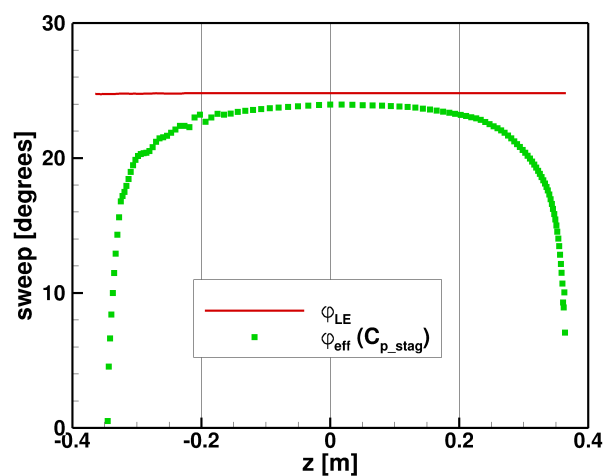

Figure 4: Leading edge sweep angle (red line) and effective sweep angle (green symbols) as a function of spanwise position $z$.

tion the pockets span a total of $680 \mathrm{~mm}$, but a metallic strip of $5 \mathrm{~mm}$ width, which contains the pressure taps, separates the TSP area in two parts. The model has 50 pressure taps (diameter of $0.2 \mathrm{~mm}$ ) in total. To monitor the temperature difference between model and flow during the measurement, four type $\mathrm{K}$ thermocouples (Omega SRTC-TT-KI-40) are installed $0.8 \mathrm{~mm}$ beneath the TSP surface. The thermocouple signals are recorded with an acquisition frequency of $1.6 \mathrm{~Hz}$.

After application of the TSP into the pockets, the TSP and metallic surface were polished to a mirror-like finish (maximum roughness height $R_{z}=$ $0.33 \mu \mathrm{m}$ ). Furthermore, the interfaces between TSP and aluminum were treated to achieve only small imperfections, i.e., gaps smaller than $200 \mu \mathrm{m}$ with an average depth of $5 \mu \mathrm{m}$.

On the suction side the transition was fixed at $x / c=0.05$ by means of zig-zag tape to secure comparability.

\subsubsection{Surface conditions}

The area upstream of the TSP coating, i.e., upstream of $x / c=0.12$, was used to vary the surface condition. Three different surface conditions were tested: highly polished (average $R_{z}=0.33 \mu \mathrm{m}$ ), unpolished (average $R_{z}=4.5 \mu \mathrm{m}$ ), and the highly polished surface with applied discrete roughness elements (DRE).

The DRE were spray-coated onto the highly polished aluminum surface using a stencil, which prescribes the specified diameter for the roughness elements and their spanwise distance. This application technique is different to previous experiments in which rub-on dots were utilized Hunt and Sarid (2011); Radetzsky et al. (1999). For the current test

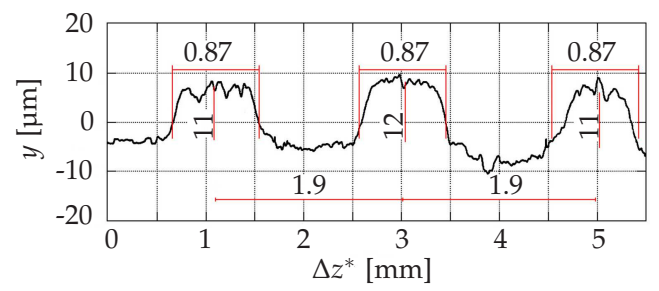

Figure 5: Tactile profile measurement of discrete roughness elements (DRE).

the DRE were placed at $x / c=0.03$, i.e., at the neutral point of CF instability.

The resulting shape of the DRE was measured directly on the model with a tactile profilometer (Perthometer S2, Mahr GmbH) with an accuracy of \pm $8 \mathrm{~nm}$ and is shown in figure 5 exemplarily for three adjacent DRE. The measurement was performed parallel to the leading edge, for this reason the spanwise coordinate is $z^{*}$. For all applied DRE an average height of $h \approx 11.8 \mu \mathrm{m}$, diameter of $\varnothing \approx 0.88 \mathrm{~mm}$, and an average distance between the centers of the DRE of $\Delta z \approx 2 \mathrm{~mm}$ was measured. The spanwise spacing of the DRE generates a wavenumber of $\gamma^{*} \approx 3450$.

\subsection{Temperature-sensitive paint method}

In the current experiment a TSP Ondrus et al. (2015) with high temperature sensitivity and high emission of fluorescent light was used. The TSP setup consisted of two LEDs (IL-105/6X Illuminator, HARDsoft) and a scientific black-and-white camera. During the measurement two types of cameras were used: a scientific 14-bit CCD camera (PCO.4000, PCO AG) and a 12-bit CMOS camera (FASTCAM SA1, Photron). The CCD camera was used to perform TSP measurements of the entire model with $0.5 \mathrm{~Hz}$ acquisition frequency and a spatial resolution of $0.2 \mathrm{~mm} / \mathrm{px}$ and the CMOS camera was used for time-resolved TSP visualization of a detail of the model with $1 \mathrm{kHz}$ acquisition frequency and a spatial resolution of $0.3 \mathrm{~mm} / \mathrm{px}$.

The equipment was arranged at the test section of the LWT as illustrated in figure 6. Both cameras share the location, i.e., perpendicular to the model. One LED continuously illuminates the TSP from upstream and downstream, respectively. The camera and LEDs were equipped with appropriate filters, i.e. a low-pass filter (cut-off wavelength $550 \mathrm{~nm}$ ) in front of the LEDs and a bandpass filter $(630 \mathrm{~nm}$ central wavelength and $75 \mathrm{~nm}$ band width) in front of the cameras to separate fluorescence light from excitation 


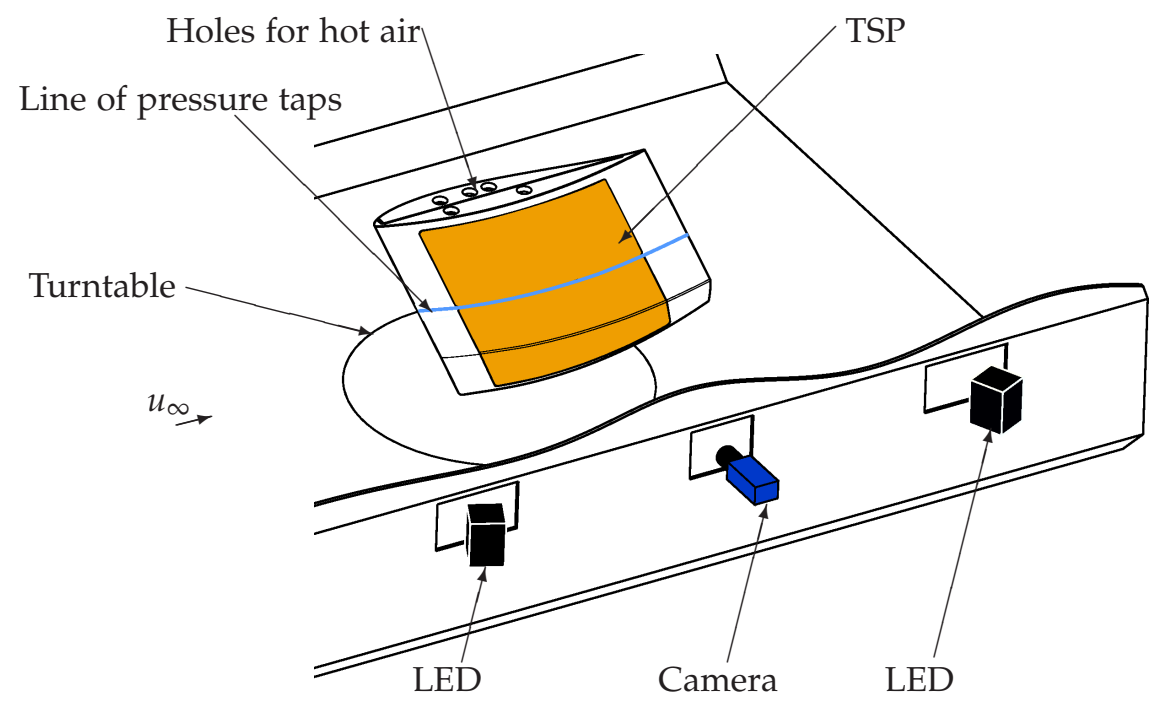

Figure 6: Isometric view of test section with the wind tunnel model and the TSP setup. The top wall is omitted.

and ambient light.

In this experiment the necessary heat flux between model surface and fluid was provided by blowing hot air through the hollow wind tunnel model. Four constant power heat blowers were used to generate the hot air; the hot air flows through the model from below, see figure 6 This setup is very robust but it does not allow to control the temperature difference between model surface and fluid. So the heat flux cannot be adapted to fluid temperature changes, which occure due to the intake of the LWT being in nature.

The main challenge for the TSP method is caused by the design of the LWT. The test section cannot be darkened because of the open return without shielding by a building. Therefore, the amount of background light in the test section depends on the sun's position and the weather. The walls of the test section were covered with black foil to reduce the amount of stray light reaching the test section from outside. Because the amount of background light remained significant despite the measures taken, the time to acquire one data point, i.e., the time between starting the measurement of the reference images and finishing the last run image, had to be kept short to avoid changes of the background light. Furthermore, the amount of fluorescent light was maximized by maximizing the excitation light from the LEDs. The LEDs provide a light power of approximately $3.5 \mathrm{~W}$ each at maximum LED current. With these settings the camera exposure time could be set to $120 \mathrm{~ms}$ for the PCO.4000 camera and to $1 \mathrm{~ms}$ for the SA1 camera.

\subsubsection{Data acquisition}

The data were acquired by first setting the angle of attack. Then, the heating was started. When the thermocouples showed that the model had reached nearly constant temperature, the so called dark images and wind-off images were taken. The dark images are acquired without excitation light and are used to quantify the background light. The wind-off images capture the temperature distribution in the absence of flow. Afterwards, the wind tunnel was started and for all desired Reynolds numbers (e.g. for the DRE investigation (see section 3.3): $\operatorname{Re}_{c}=$ 4.4, 4.6, 4.8, 5.0 $10^{6}$ ) 30 images, which are called windon images, were acquired.

In the evaluation the average of the acquired 30 dark images is subtracted from the averaged wind-off and wind-on image to reduce the influence of background light. Finally the averaged wind-off image is divided by the averaged wind-on image to obtain the TSP visualizations. These gray values are a function of the surface temperature difference between windoff and wind-on conditions. The temperature difference depends on the local skin friction, caused by the flow around the wind tunnel model. The final step of the evaluation is to use the markers on the model to map the TSP results from the two-dimensional camera plane onto a three-dimensional surface grid Klein et al. (2005).

From the gray values on the surface grid the location of laminar-turbulent transition can be derived at the location of largest gradient Lemarechal et al. (2019). The measured gray values are spatially me- 
dian filtered in flow direction. Afterwards, the derivative of the gray values in streamwise direction is computed. The transition is detected at the minimum of this function. Due to the wind-tunnel model's internal structure causing large gradients of the gray value distribution, an automated evaluation was not possible.

\subsection{Linear stability calculation}

In addition to the stability calculations performed in the design process of the wind tunnel model, the pressure distributions $C_{p}$ acquired during the wind tunnel testing were fed in an automated stability calculation tool in a post-processing step. Thus, effects of the actual wind tunnel conditions and deviations from the simulated $C_{p}$ distributions, e.g. due to the applied roughness elements, could be captured. The stability properties of the boundary layer are determined in three steps, assuming infinite swept-wing flow and neglecting curvature effects.

The measured $C_{p}$ distribution is pre-processed and the attachment line and the boundary layer edge velocity are determined. The boundary-layer profile at the stagnation line is construced based on the incompressible equations for a two-dimensional FalknerSkan boundary layer White and Corfield (1991), being identical to a Hiemenz stagnation line flow for the Hartree parameter $\beta_{H}=1.0$, and the spanwise component based on the Cooke solution Cooke (1950).

A boundary-layer integration based on compressible parabolized Navier-Stokes equations is performed, yielding the input for the stability solver.

The stability properties are calculated with a matrix solver, where the equations of motion are linearized and parallel flow is assumed. Complete spectra of the complex spatial wavenumber $\alpha=\alpha^{*}+\mathrm{i} \alpha_{i}$ are computed, defined perpendicular to the leading edge. Stationary crossflow modes are investigated for the spanwise-wavenumber range $\gamma^{*}$ shown in figure 3 at frequency $\omega=0$, two-dimensional TollmienSchlichting waves are also analyzed at $\gamma^{*}=0$. Further details on the stability solver are given bv Kurz and Kloker (2014).

\section{Results AND Discussion}

At first a result for the highly-polished leading-edge surface is presented, see section 3.1. In section 3.2 the heat flux between fluid and model was varied for the same surface condition to investigate whether the result for the chosen heat flux settings are similar to the adiabatic case. The influence of the heat flux is discussed for a test case with moderate amplification of CF instability at $\operatorname{Re}_{c}=3.77 \cdot 10^{6}$ and $\alpha=6^{\circ}$. Finally, results with an altered leading edge, i.e. with DRE (section 3.3) and the unpolished leading edge (section 3.4), are presented. The DRE are investigated in the Reynolds number range of $3.77 \cdot 10^{6} \leq \operatorname{Re}_{c} \leq$ $5.00 \cdot 10^{6}$ and fixed angle of attack $\alpha=6^{\circ}$.

\subsection{Highly polished leading edge}

The configuration with the highly polished leading edge is the reference configuration of the current test. In figure 7 the TSP visualization for $\operatorname{Re}_{c}=5 \cdot 10^{6}$ at an angle of attack $\alpha=6^{\circ}$ is presented in the uncorrected view of the TSP camera. This figure also defines the $x-z^{*}$ coordinate system used to present the remaining TSP visualizations. The $x$-axis is aligned with the flow direction and $z^{*}$ is parallel to the leading edge. The gray values used to present the TSP visualizations can be interpreted in the following way: Darker areas correspond to higher heat transfer, which can be caused by turbulent flow, and the brighter areas correspond to lower heat transfer, i.e. laminar flow. The typical sawtooth pattern of CF-induced transition is visible in figure 7 The spanwise extent of the regular sawtooth pattern is approximately one third of the model span. This matches the design goals to achieve quasi-infinite swept-wing flow in the middle third of the model as discussed in section 2.2. The region of quasi-infinite swept-wing flow as seen in the TSP result is in good agreement with the predicted spanwise extend of constant effective sweep angle as can be seen in figure 4 Below the line of pressure taps one turbulent wedge begins significantly further upstream than the sawtooth pattern. It is expected that this turbulent wedge is triggered by a pressure tap close to the indifference point, which increases the initial amplitude of the CF modes locally.

The structural design of the wind tunnel model resulted in an inhomogeneous distribution of the heat capacity. This inhomogeneous distribution of the heat capacity causes variations of the surface temperature distribution independent of the flow phenomena. The light-gray grid structure parallel to the leading edge and the line of pressure taps visible in figure 7 is the result of the spars and stringers, see section 2.2. Another drawback of the model design is the high heat conductivity of the aluminum, which reduces the contrast of fine structures caused by the flow. For compensation a large difference between model surface temperature $T_{m}$ and flow temperature 


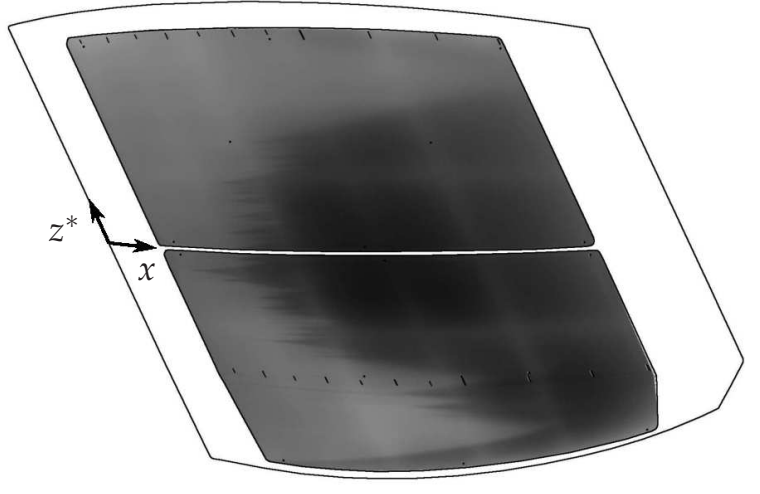

Figure 7: TSP visualization of laminar-turbulent transition. The entire model, as seen by the TSP camera, is shown. $R e_{c}=5 \cdot 10^{6}$ at an angle of attack $\alpha=6^{\circ}$. Lines placed every $5 \%$ of the chord up to $x / c=0.5$ and every $10 \%$ further downstream. The flow direction is from left to right.

$T_{f}$ was used to achieve sufficient contrast for the flow structures. Possible influence of the heating on the transitional flow is discussed in section 3.2

A clear influence of the wind tunnel walls can be seen in the top third of the model, where the extent of laminar flow is significantly increased over the entire TSP area. At the bottom side of the model the laminar flow reaches also further downstream than in the region of crossflow induced transition in the middle third of the model. This increased laminar flow is caused by the reduced effective sweep angle, which leads to weaker CF instability. The twist of the model causes a turbulent wedge, which begins at $x / c \approx 0.4$. Another turbulent wedge is visible close to the bottom of the model. It is starting near the leading edge and is triggered by the turbulent boundary layer on the tunnel walls.

Figure 8 shows a detail from figure 7 in the designated coordinate system with the flow from left to right. It can be seen that the TSP method also resolves the footprint of the stationary crossflow vortices, i.e., parallel lines leading up to the turbulent flow can be seen. The spacing of the footprints is interpreted as the dominant stationary $\mathrm{CF}$ wavelength $\lambda$. The visible footprint of the $C F$ vortices varies from vortex to vortex, which is interpreted as a variation in vortex strength. An increase of the vortex strength along its axis is however barely visible. This leads to the conclusion that the $\mathrm{CF}$ vortices are only visible in the nonlinear regime, when the growth is (almost) saturated. As can be expected, strong vortices,

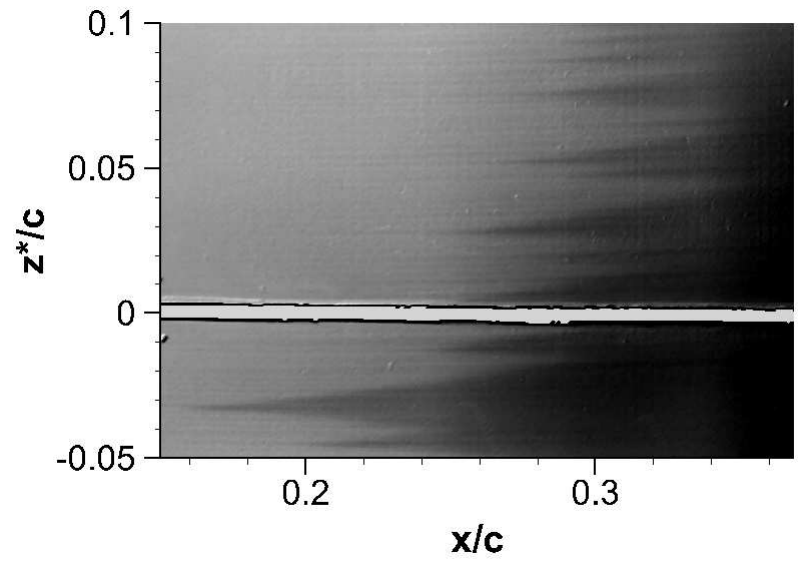

Figure 8: Close up view of laminar-turbulent transition location. The flow direction is from left to right.

i.e. with especially dark footprint, lead to the onset of the turbulent wedges. Note also that the vortex next to the one leading to the wedges is also stronger than the surrounding ones. The apex of the turbulent wedges is located inside of the footprint of a CF vortex. This is different from the observations with sublimating chemicals where the apex of the turbulent wedge was detected between the footprint of the CF vortices Kohama et al. (1996). In contrast investigations with hot-wire anemometry showed that the apex of the turbulent wedges is located at the location of the vortices Kohama et al. (1996). From the observations in this experiment it is concluded that the TSP method visualizes the same transition location as a hot-wire would.

The early onset of transition at $z^{*} / c=-0.032$ is probably caused by a particularly large event in the surface texture at the leading edge region, which provides locally a large initial amplitude for the CF instability. The structure inside of the turbulent wedge shows a continuation of the vortex footprint into the turbulent area as indicated in figure 8 . This behavior was also observed with DNS Wassermann and Kloker (2002) and in experiments with sublimating chemicals Dagenhart et al. (1989).

\subsection{Heating variation}

The heating setup of hot air blowers with constant electrical power does not allow to adapt the temperature difference between the model surface and the fluid. This leads to an average over-temperature ratio of $T_{m} / T_{f}=1.07$ with a standard deviation of 0.08 . Only three previous studies related to this topic are available: In experiments it was found that the 


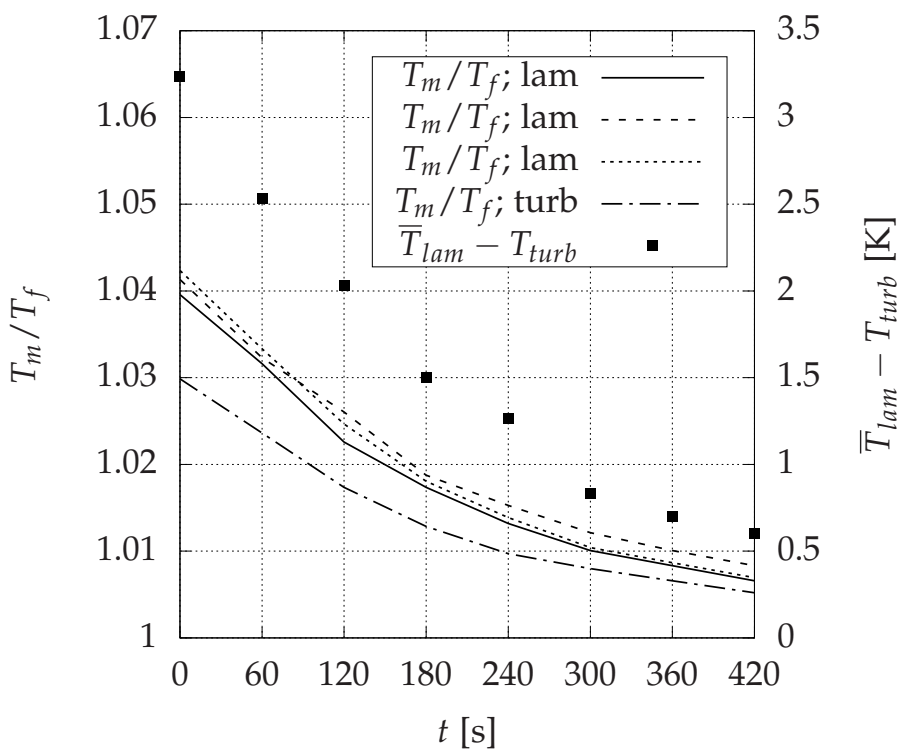

Figure 9: Evolution of over-temperature ratio $T_{m} / T_{f}$ and temperature difference between laminar $\bar{T}_{\text {lam }}$ and turbulent $T_{\text {turb }}$ flow during cool down.

boundary layer is destabilized by a two-dimensional heating strip at the leading edge and earlier laminarturbulent transition occurs for $T_{m} / T_{f} \approx 1.21$ Dovgal et al. (1990). In numerical investigations for crossflow instability at transonic speeds with a cooled wall flow stabilization is found, see, e.g., Lekoudis (1980); Friederich (2013). The influence of the model heating on the detected phenomena was therefore investigated.

At first, one data point with the standard heating power settings was acquired. For the weather conditions at that time resulted in $T_{m} / T_{f} \approx 1.04$. Afterwards, the heating was switched off and every $60 \mathrm{~s}$ TSP images and the absolute model were acquired to investigate the influence of the temperature ratio $T_{m} / T_{f}$. In figure 9 the decreasing temperature ratio between model and flow is shown for the four thermocouples on the side under investigation. For the chosen flow conditions, i.e., $\alpha=7^{\circ}$ and $\operatorname{Re}_{c}=4.19 \cdot 10^{6}$ three thermocouples were located underneath laminar flow and one was located underneath turbulent flow. As expected the model temperature decreases exponentially and the surface temperature difference between laminar $T_{l a m}$ and turbulent $T_{\text {turb }}$ flow diminishes, which characterizes the contrast of the TSP visualizations.

In figure 10 the TSP visualizations for two temper-
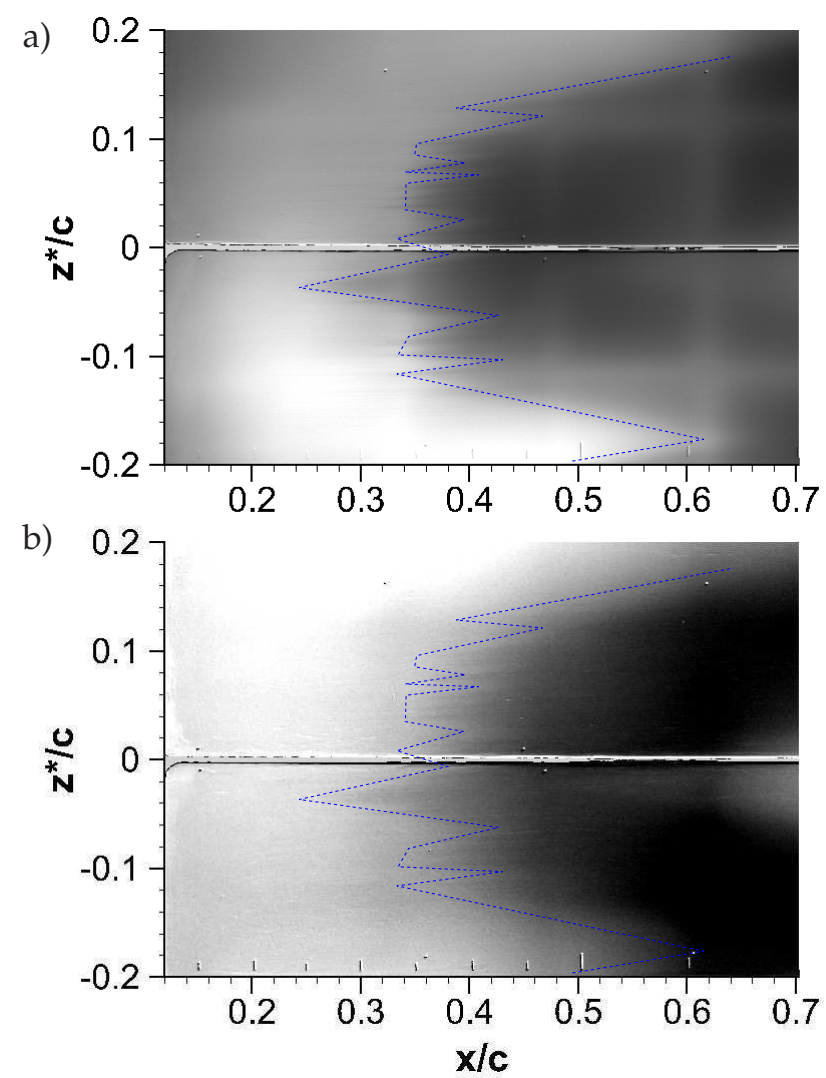

Figure 10: Transition location for $\alpha=7^{\circ}$ and $\operatorname{Re}_{c}=4.19 \cdot 10^{6}$ with a temperature ratio of $T_{m} / T_{f} \approx 1.04$ a) and $T_{m} / T_{f} \approx 1.01 b$ ). The blue line indicates the location of the laminar turbulent transition as derived from a), which is also shown in $b$ ). The flow direction is from left to right. 
ature ratios $T_{m} / T_{f} \approx 1.04$ and 1.01 is shown. The gray scale is adapted for both visualizations individually to increase the contrast. Footprints of crossflow vortices and the vortices becoming turbulent can be observed in figure 10 a). However, the lower temperature ratio (figure 10 b)) shows a significantly worse contrast and fine structures cannot be observed anymore. Nevertheless, the laminar-turbulent transition as indicated by a large change of gray scale values is hardly changed between the two temperature ratios.

Considering the surface temperature difference between laminar and turbulent flow it is obvious that the TSP method showed better contrast for $T_{\text {lam }}-$ $T_{\text {turb }}=3.3 \mathrm{~K}$ as in figure $10 \mathrm{a}$ ). When the temperature difference decreases (see figure 9 the contrast decreases and at $T_{\text {lam }}-T_{\text {turb }}=0.8 \mathrm{~K}$ (figure $10 \mathrm{~b}$ )) the contrast reaches a limit for practical applications. The laminar-turbulent transition could not be visualized for smaller temperature differences. For wind tunnel models without a pronounced pattern of the heat capacity (grid pattern in figure 7) and a lower heat conductivity than aluminum, the limit will be smaller than $T_{\text {lam }}-T_{\text {turb }}=0.8 \mathrm{~K}$. This would reduce the necessary temperature difference between model and fluid significantly. Temperature differences similar to those used in previous experiments to investigate crossflow induced transition of $T_{m}-T_{f} \approx 5 \mathrm{~K}$ can be achieved Crawford et al. (2013).

The final conclusion is that an influence of the nonadiabatic surface conditions on the flow could not be observed for $T_{m}-T_{f} \leq 1.04$. This was investigated by switching of the heating power while continuing with the TSP acquisition. However, the development of integrated electrical heating, e.g. carbon nanotubes as introduced by Klein et al. (2014) and current carrying carbon fibers Petzold and Radespiel (2015), and wind tunnel models with homogenous heat capacity distribution and low heat conductivity are vital for improving the quality of TSP results.

\subsection{Discrete roughness elements}

The TSP method was also used for investigating spray-coated DRE at various chord Reynolds numbers and at fixed angle of attack $\left(\alpha=6^{\circ}\right)$. A detail of the TSP result, which is directly comparing the transition location of the clean configuration and the transition delayed via DRE, is shown in figure 11 a) and $11 \mathrm{~b})$, respectively. Two prominent differences are visible: delay of the transition location (indicated with the solid white line) and a stronger footprint of the stationary $\mathrm{CF}$ vortices with a larger spacing and

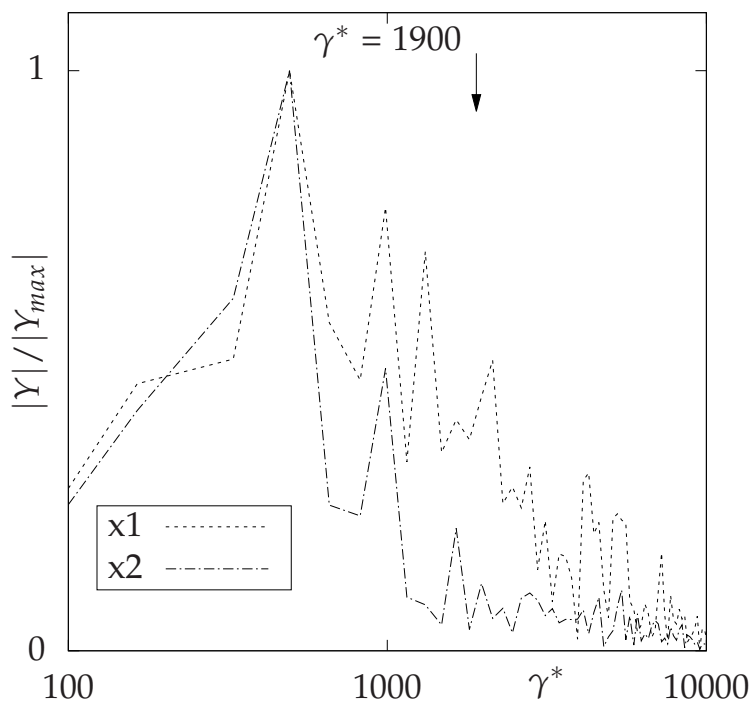

Figure 12: Amplitude spectrum versus wavenumber for the case without DRE. The spectrum is derived from the TSP cuts as indicated in figure 11a).

earlier beginning with DRE, cf. 11b).

From the TSP data of figure 11 a) and 11 b) the wavenumber spectrum of the stationary $\mathrm{CF}$ vortex footprints was calculated by Fourier analysis at five different locations $x 1$ and $x 2$ (figure 12) and $x 3, x 4$, and $x 5$ (figure 13). Starting with the clean case (figure 11 a) the visualization shows hardly any visible structures upstream of $x / c=0.3$ but the wavenumber spectrum in figure 12 shows several peaks including the expected wavenumber of $\gamma^{*}=1900$ at the $x 1$ location. Further downstream the transition starts in CF vortices with a distance of $\gamma^{*} \approx 500$ (figure 11 a) at $x / c=0.35(\mathrm{x} 2))$, which is smaller than the predicted naturally most amplified wave-number. The transition is located at $x / c \approx 0.4 \pm 0.02$.

When the UFD mode $\left(\gamma^{*}=2850\right)$ is triggered by the DRE, this is visible in the wavenumber distribution ( $x 3$ in figure 13) and also in the visualization (figure $11 \mathrm{~b}$ )), where an evenly spaced vortex footprint is already visible at $x / c \approx 0.21$. Additionally to the triggered wavenumber mode, a low-wavenumber mode $\left(\gamma^{*} \approx 850\right)$ appears. Note that further downstream this wavenumber remains of significant impact on the footprint. Closer to the transition location at $x 4$ the naturally most amplified wavenumber $\left(\gamma^{*}\right.$ $=1900$ ) appears. The UFD mode delays transition to $x / c \approx 0.5 \pm 0.02$ for $\operatorname{Re}_{c}=5.0 \cdot 10^{6}$.

The test showed a linear variation of the transition 
a)

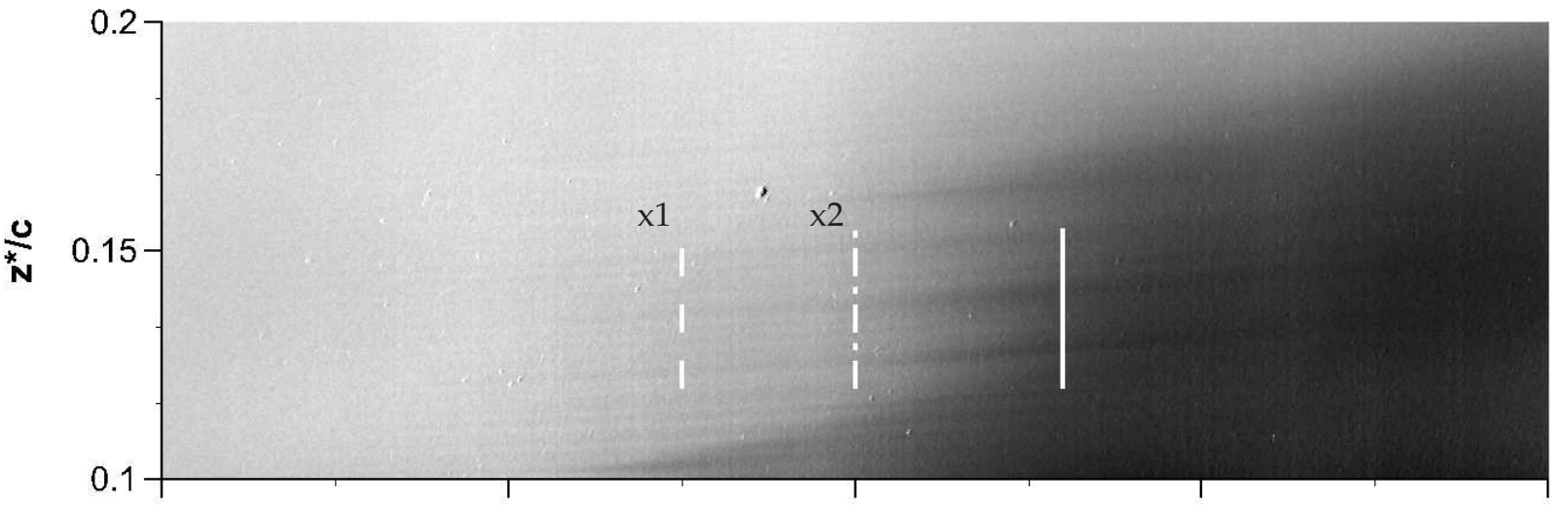

b)

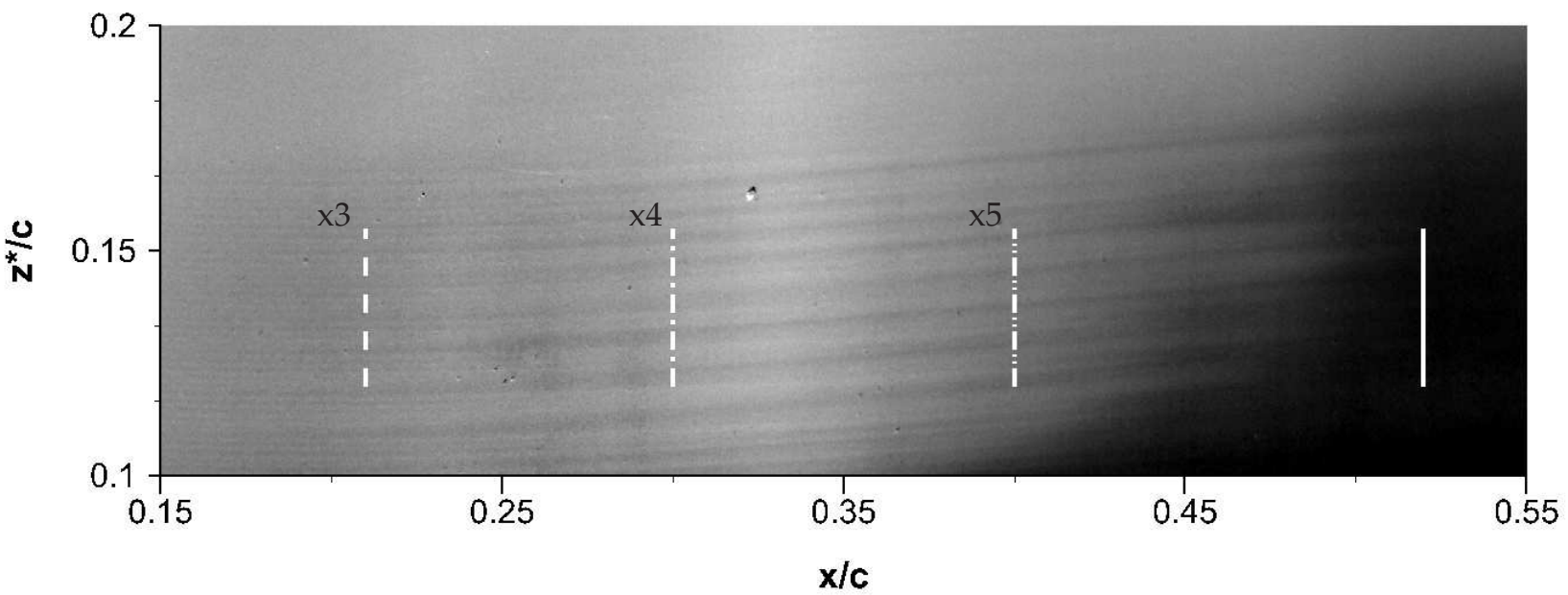

Figure 11: TSP visualization for $R e_{c}=5.0 \cdot 10^{6}$ and $\alpha=6.0^{\circ}$ of polished surface a) in comparison to the polished surface with spray coated DRE b). The solid lines mark the transition location and at the dashed lines the amplitude spectrum of the wavenumber is derived. The flow direction is from left to right. 


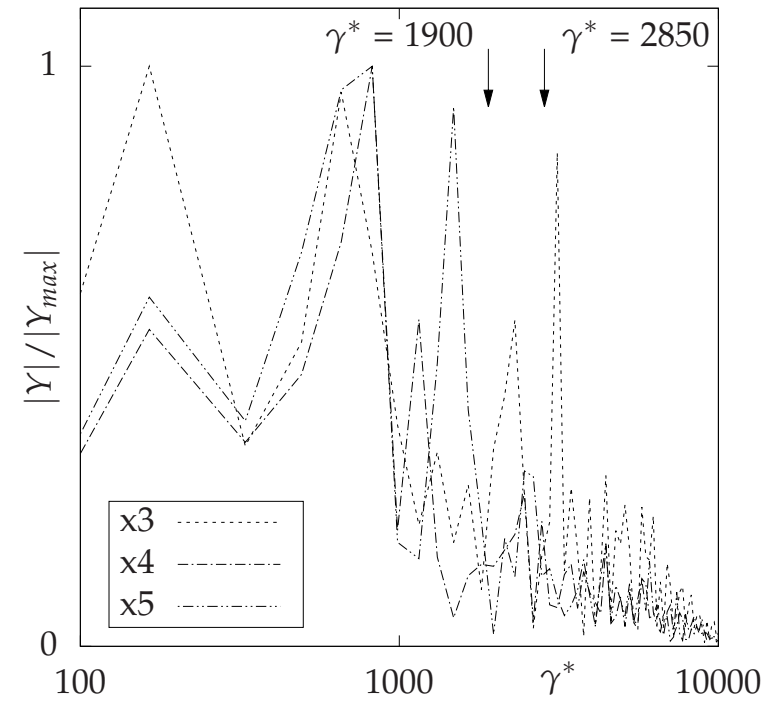

Figure 13: Amplitude spectrum versus wavenumber for the case with DRE. The spectrum is derived from the TSP cuts as indicated in figure $11 \mathrm{~b}$ ).

\begin{tabular}{cccc}
\hline $\mathrm{Re}_{c} \cdot 10^{6}$ & $x_{t} / c$ no DRE & $x_{t} / c$ DRE & $\Delta$ LAM [\%] \\
\hline 4.42 & 0.70 & 0.57 & -18.6 \\
4.61 & 0.56 & 0.52 & -7.2 \\
4.80 & 0.46 & 0.51 & 10.8 \\
5.00 & 0.41 & 0.52 & 26.7 \\
\hline
\end{tabular}

Table 1: Transition variation by DRE for different chord Reynolds numbers at $\alpha=6^{\circ} . \triangle L A M$ is the increase of laminar flow due to application of the DRE method; negative values correspond to premature transition.

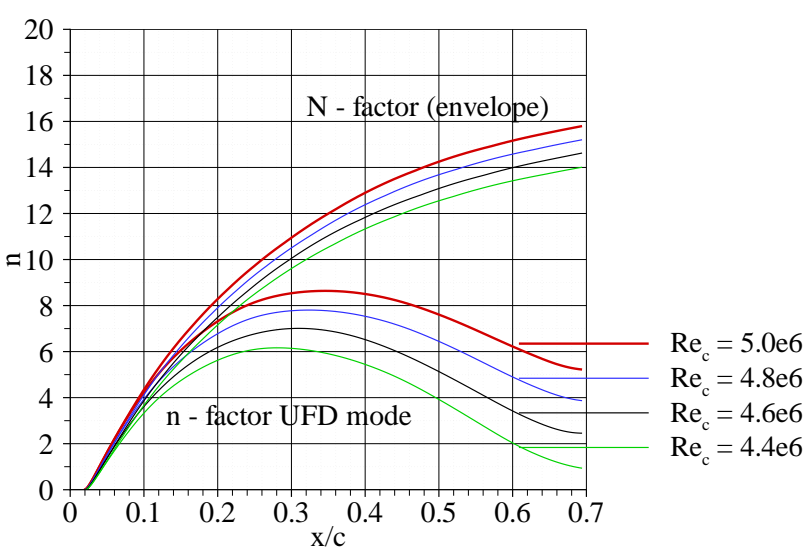

Figure 14: N-factor diagram of the envelope and UFD mode.

location with increasing Reynolds number, when no DRE are applied, see table 1. Transition delay sets in for $\operatorname{Re}_{c} \geq 4.8 \cdot 10^{6}$ (see table 1). In figure 14 it can be seen from the linear stability calculations that the UFD mode reaches higher $\mathrm{n}$-factors (and thus amplitudes). However, the n-factor of the UFD modes remain, for the investigated Reynolds number range, below $n \leq 10$ and it is expected that this is below the critical N-factor. When transition is successfully delayed the introduced UFD mode is detectable in the TSP result at $x / c \approx 0.21$ (figure 11) but the mode leading to transition has a wavenumber of $\gamma^{*} \approx 850$, which was not observed in the clean case.

The successful delay of the laminar-turbulent transition could only be achieved at the spanwise edge of the infinite-swept wing flow, where the CF instability is less amplified due to the reduced effective sweep angle (figure 44). At $\operatorname{Re}_{\mathcal{c}}<4.7 \cdot 10^{6}$ the transition of the clean configuration is so far aft, that introducing the UFD-mode causes premature transition. Introducing the DRE leads to an increased initial amplitude of the CF instability compared to the highly polished leading edge surface without DRE. This leads to premature transition for $\operatorname{Re}_{c}<4.7 \cdot 10^{6}$.

Larger amplitudes of the stationary-crossflow disturbance can be observed, which can be concluded from the earlier detectability of the footprint of the stationary $\mathrm{CF}$ disturbance.

\subsection{Unpolished leading edge}

Figure 15 shows the TSP visualization of the model with a rough leading edge at $\operatorname{Re}_{c}=3.8 \cdot 10^{6}$ and $\alpha=9^{\circ}$. During the experiments an interesting phenomenon was observed: When the RMS was calcu- 


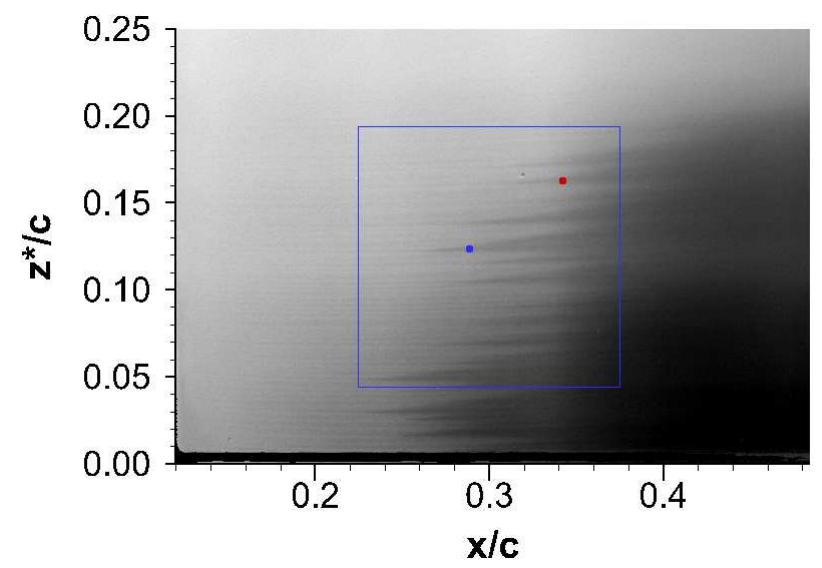

Figure 15: Transition location generated by an unpolished surface. The blue square indicates the field of view of the high-speed camera and the artificial blue and red dot tag two specific vortices hereafter.

lated for each pixel from a time-series of 30 run images the onset of some turbulent wedges showed significantly increased root mean square (RMS) values, see figure 16. In the RMS result brighter areas indicate larger RMS values, i.e., regions of larger variations. The RMS results indicate that the onset of the turbulent flow, which is forming the saw tooth pattern, varies significantly during the measurement time. This behavior was observed only for configurations with an unpolished leading edge. In figure 16 the spatial distribution of the RMS value is shown. An area of very large RMS value is visible between $z^{*} / c=0.13$ and $z^{*} / c=0.17$ in the vicinity of the red dot, and the upstream end of this region shows the sawtooth pattern, which is typical for crossflow instability, see figure 15, Furthermore, a small region of increased RMS is visible between $z^{*} / c=0.05$ and $z^{*} / c=0.10$. The rest of the field of view in figure 16 shows low RMS values, e.g., in the vicinity of the blue dot.

When the RMS results of figure 16 are compared with the standard TSP visualization, which is shown in figure 15, it can be observed that the increased RMS is located in the beginning of some turbulent wedges. However, not necessarily all turbulent wedges show increased RMS values. Comparing two turbulent wedges, one with increased RMS (indicated with a red dot) and one without increased RMS (indicated with a blue dot), in figure 15, no difference between these turbulent wedges is visible.

To further investigate this unsteadiness, the data point was repeated with the high-speed camera and again the RMS of each pixel was calculated, see figure

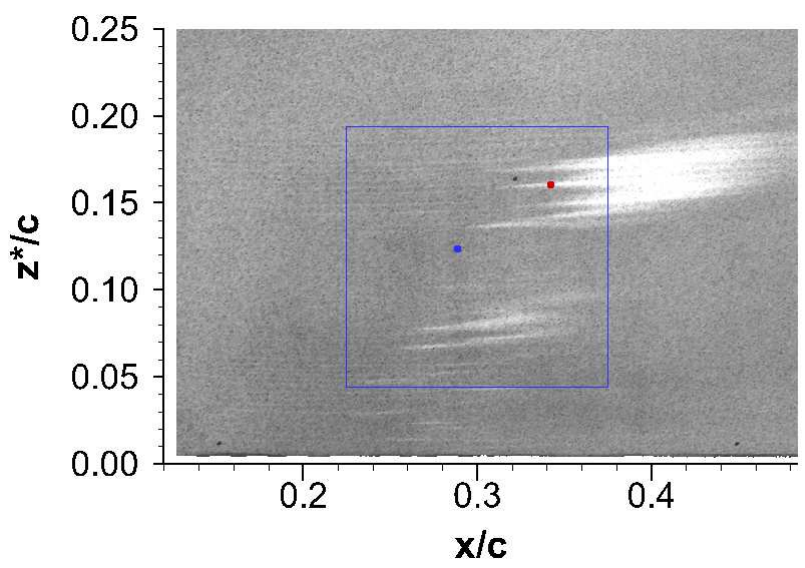

Figure 16: RMS of the TSP visualization shown in figure 15 Brighter gray values indicating larger RMS value and the blue square indicates the field of view of the high-speed camera.

17 The field of view of the high-speed camera was limited; therefore, not sufficient markers were visible to map the results onto the model grid. By using image based data data reduction, the red and blue markers indicated approximately the same locations as the results in figure 16 and figure 15 Between recording the results of figure 16 and figure 17 the wind tunnel was stopped, the experimental setup was adapted to the high-speed camera measurement, and the wind tunnel was restarted. However, the results of both cameras agree very well.

The increased acquisition rate with the high-speed camera allows to observe the behavior of the laminarturbulent transition in more detail and the cause of the locally increased RMS can be investigated. In the time-series a back and forth motion in flow direction of the apex of the turbulent wedge can be observed in the regions of increased RMS. However, a change of the most dominating wavenumber is not visible. Figure 18 compares the temperature-dependent $(f(T))$ intensity ratio of a turbulent wedge with a steady location of transition onset (indicated with the blue dot in figure 17) with a turbulent wedge with a highly dynamic location of transition onset (indicated with the red dot in figure 17. From the time-resolved measurement the variation of the temperature-dependent $(f(T))$ intensity ratio over time is shown. A lowfrequency movement without any dominating frequency along the vortex axis is visible for the unsteady wedge. The onset of the wedge marked with the red symbol moves $44 \mathrm{~mm}(\Delta x / c=0.044)$ in flow direction during the measurement time.

A significant movement of the onset of turbulent 


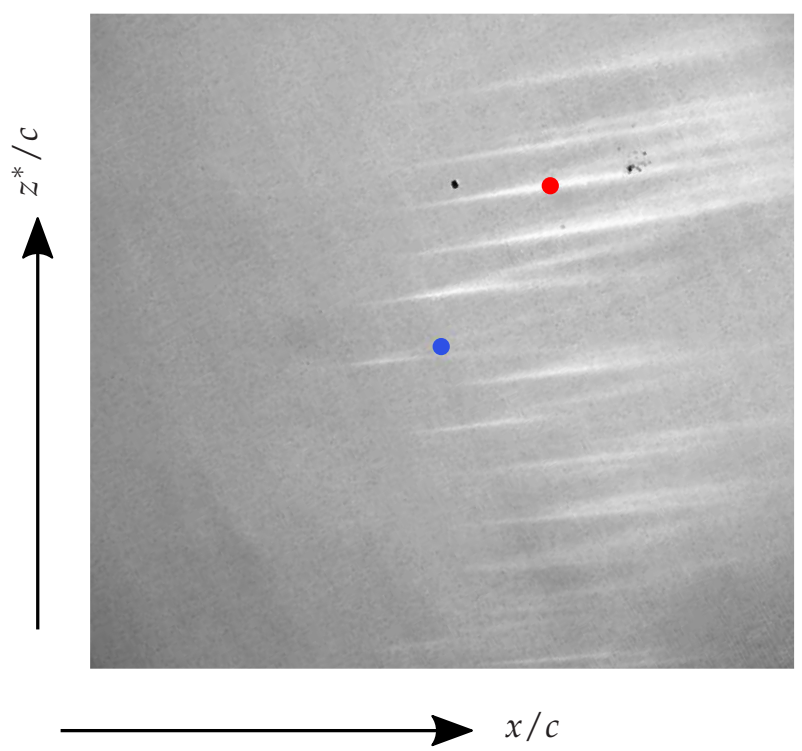

Figure 17: RMS of a time-resolved acquisition with a high-speed camera.

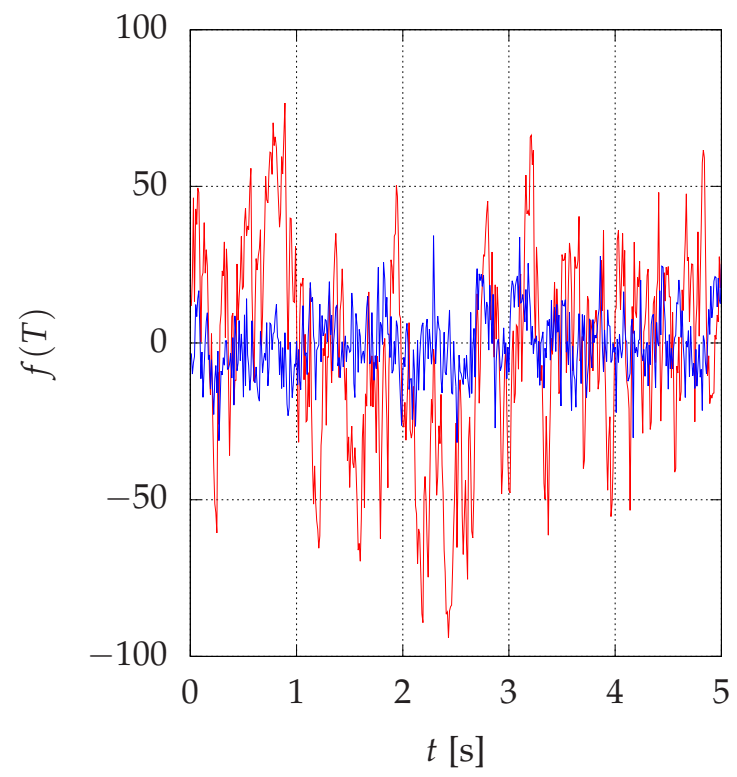

Figure 18: Time series of the intensity ratio $(f(T))$ of a stationary crossflow vortex with unsteady (red) and steady (blue) transition onset, respectively. wedges was only observed when the leading edge surface was unpolished and therefore very rough. The movement of the wedges seems to be independent for each wedge indicating that a local mechanism causes this variation. The unpolished surface seems to feed a significant initial amplitude to many different wavenumbers. Due to the unpolished surface, the characteristics of the surface varies for different spanwise locations, so the initial amplitude is in addition depending on the spanwise location. In combination with small time-dependent fluctuations, this could set up a scenario similar to the one discussed in section 3.3. where the flow deformation due to a particular mode leads to an influence (e.g., damping) on another mode. Finally, this could lead to the observed variation of the onset of turbulent wedges. In line, certain surface conditions seem to be more prone to excite a dynamic behavior of the transition onset.

\section{Conclusions}

In this experiment the capabilities of the TemperatureSensitive Paint (TSP) method to investigate laminartur-bulent transition dominated by crossflow instability were explored. Various conditions of the leading edge surface, i.e., highly polished, unpolished, and highly polished with discrete roughness elements (DRE), were investigated. For this experiment, a swept-wing wind tunnel model for the investigation of stationary crossflow instability was designed for the Laminar Wind Tunnel of the University of Stuttgart. The model provides a quasi-infinite sweptwing flow in the middle third of the test section with a simple geometry.

The TSP method was used to study laminarturbulent transition, which was induced by stationary crossflow instability. Besides the transition location, also the footprints of the stationary crossflow vortices were visualized.

The influence of the model heating on the transition location was investigated to check for a possible influence of the measurement technique on the flow. In the investigated temperature range of $T_{m} / T_{f} \leq 1.04$ no influence on the laminar-turbulent transition was observed. For small temperature difference the contrast of the visualization decreased significantly. When the surface temperature difference between laminar and turbulent flow decreases below $T_{\text {lam }}-T_{\text {turb }}=0.8 \mathrm{~K}$ the contrast becomes insufficient because of the strong temperature signatures from the model itself.

In the case of a highly-polished leading-edge sur- 
face, transition was successfully delayed for Reynolds numbers $\operatorname{Re}_{c} \geq 4.8 \cdot 10^{6}$ by applying the upstream flow deformation (UFD) method based on DRE. From the experimental data, the wavenumber was derived and compared to linear stability calculations. Good agreement between the predicted wave numbers from the linear stability calculations and the TSP results was found.

Furthermore, high local RMS values were found for the case of an unpolished leading-edge surface. Using time-resolved TSP visualizations (acquisition frequency of $1 \mathrm{kHz}$ ) a variation of the transition onset location was observed in regions of increased RMS. It is assumed that the surface roughness causes this variation in combination with small inflow turbulence fluctations, together this leads to a recoprocal mode interaction. Since these variations were restricted to small spanwise locations and occuring repeatably at the same location, the localized surface roughness pattern must be of dominating importance for the observed behavior of the transition location.

Acknowledgements The funding of this work by the Federal Ministry for Economic Affairs and Energy (BMWi) under LuFo V-1 LDAinOp AP 1210 âĂIJLE-RauheitâĂ (Grant No. 20A1302B) is gratefully acknowledged. The authors would like to thank the designers of the initial baseline airfoil: G. Schrauf (DLR Braunschweig) and W. Kühn (former Airbus). Also, the support in the coating and surface treatment process by V. Ondrus (University of Hohenheim), C. Fuchs, D. Yorita, and A. Weiss (DLR Göttingen) is gratefully acknowledged. And finally the authors would like to thank M. Langohr-Kolb and C. Vetter (University of Stuttgart) for their support during the wind tunnel measurement.

\section{REFERENCES}

H. Bippes. Basic experiments on transition in threedimensional boundary layers dominated by crossflow instability. Progress in Aerospace Sciences, 35:363-412, 1999. doi: 10.1016/S0031-8914(53)80099-6.

J. Birkemeyer. Notes on Numerical Fluid Mechanics and Multidisziplinary Design, volume 72, chapter Adapted wind tunnel walls for swept wing experiment, pages 44-51. Springer-Verlag, 1991.

J.C. Cooke. The boundary layer of a class of infinite yawed cylinders. Mathematical Proceedings of the Cambridge Philosophical Society, 46:645-648, 1950.

B.K. Crawford, G.T. Duncan, D.E. West, and W.S. Saric. Laminar-turbulent boundary layer transition imaging us- ing ir thermography. Optics and Photonics Journal, 3:233239, 2013. doi: 10.4236/opj.2013.33038.

J.R Dagenhart, W.S Saric, M.C Mousseux, and J.P Stack. Crossflow-vortex instability and transition on a $45 \mathrm{deg}$ swept wing. In 20th Fluid Dynamics, Plasma Dynamics and Lasers Conference, Fluid Dynamics and Co-located Conferences. AIAA-89-1892, 1989. doi: 10.2514/6.1989-1892.

H. Deyhle and H. Bippes. Disturbance growth in an unstable three-dimensional boundary layer and its dependence on environmental conditions. Journal of Fluid Mechanics, 316:73-113, $1996 . \quad$ doi: 10.1017/ S0022112096000456.

A.V. Dovgal, V.Y. Levchenko, and V.A. Timofeev. LaminarTurbulent Transition. International Union of Theoretical and Applied Mechanics, chapter Boundary Layer Control by a Local Heating of the Wall, pages 113-121. Springer, Berlin, Heidelberg, 1990. doi: 10.1007/ 978-3-642-84103-3_9.

R.S. Downs III. Environmental Influences on Crossflow Instability. PhD thesis, Texas A\&M University, 2012.

U. Fey and Y. Egami. Springer Handbook of Experimental Fluid Mechanics, chapter Transition-Detection by TemperatureSensitive Paint. Springer-Verlag, Berlin, Heidelberg, 2007.

U. Fey, Y. Egami, and C. Klein. Temperature-sensitive paint application in cryogenic wind tunnels: Transition detection at high Reynolds numbers and influence of the technique on measured aerodynamic coefficients. In 22nd International Congress on Instrumentation in Aerospace Simulation Facilities, Pacific Grove, CA, 2007, 2007.

U. Fey, C. Klein, T.J. Möller, J. Pöttner, R. Radespiel, V. Ondrus, and U. Beifuß. Notes on Numerical Fluid Mechanics and Multidisziplinary Design, volume 121, chapter Investigation of Circular Cylinder Flow in Water Using Temperature-Sensitive Paint, pages 657-664. SpringerVerlag, 2013.

T. Friederich. Control of the secondary crossflow instability: Direct numerical simulation of localized suction in threedimensional boundary layers. PhD thesis, IAG, University of Stuttgart, 2013.

T. Herbert and G. Schrauf. Crossflow-dominated transition in flight tests. In 34th Aerospace Sciences Meeting and Exhibit, 15-18 January 1996, Reno, Nevada. AIAA 96-0185, 1996. doi: 10.2514/6.1996-185.

S. Herr. Experimental investigation of airfoil boundary-layer receptivity and a method for the characterization of the relevant free-stream disturbances. PhD thesis, University of Stuttgart, 2004. 
B.J. Holmes, C.J. Obara, and L.P. Yip. Natural laminar flow experiments on modern airplane surfaces. Technical report, NASA Technical Paper 2256, 1984.

L.E. Hunt and W.S. Saric. Boundary-layer receptivity of three-dimensional roughness arrays on a swept-wing. In 41st AIAA Fluid Dynamics Conference and Exhibit, 27 - 30 June 2011, Honolulu, Hawaii. AIAA 2011-3881, 2011. doi: 10.2514/6.2011-3881.

C. Klein, R. Engler, U. Henne, and W. Sachs. Application of pressuresensitive paint for determination of the pressure field and calculation of the forces and moments of models in a wind tunnel. Experiments in Fluids, 39:475-483, 2005. doi: 10.1007/s00348-005-1010-8.

C. Klein, U. Henne, W. Sachs, U. Beifuß, V. Ondrus, M. Bruse, R. Lesjak, and M. Löhr. Application of carbon nanotubes (CNT) and temperature-sensitive paint (TSP) for the detection of boundary layer transition. In 52nd Aerospace Sciences Meeting, 13-17 January 2014, National Harbor, Maryland. AIAA 2014-1482, 2014. doi: 10.2514/6.2015-1558.

Y. Kohama, W.S. Saric, and J.A. Hoos. A high-frequency, secondary instability of crossflow vortices that leads to transition. In Proc. R. Aerosp. Soc. Conf. Bound.-Layer Transit. Control Conf. Peterhouse College, Cambridge, UK, pages 4.1-4.13, 1991

Y. Kohama, T. Onodera, and Y. Egami. Design and control of crossflow instability field. In P.W. Duck and P. Hall, editors, IUTAM Symposium on Nonlinear Instability and Transition in Three-Dimensional Boundary Layers, pages 147-156. Springer Netherlands, 1996. doi: 10.1007/978-94-009-1700-2_14.

H.B.E. Kurz and M.J. Kloker. Receptivity of a sept-wing boundary layer to micron-sized discrete roughness elements. Journal of Fluid Mechanics, 755:62-82, 2014. doi: $10.1017 / \mathrm{jfm} .2014 .425$.

S.G. Lekoudis. The stability of the boundary layer on a swept wing with wall cooling. AIAA Journal, 18:10291035, 1980. doi: 10.2514/6.1979-1495.

J. Lemarechal, C. Klein, U. Henne, D.K. Puckert, and U. Rist. Detection of lambda- and omega-vortices with the temperature-sensitive paint method in the late stage of controlled laminar-turbulent transition. Experiments in Fluids, 60:91:1-14, 2019. doi: 10.1007/s00348-019-2734-1.

T. Liu and J. P. Sullivan. Pressure And Temperature Sensitive Paints. Springer-Verlag, 2005.

B. Müller and H. Bippes. Fluid Dyn. Three-Dimens. Turbul. Shear Flows Transit, chapter Experimental study of instability modes in a three-dimensional boundary layer, pages 13.1-13.15. AGARD CP 438, 1989.
P. Nitschke-Kowsky and H. Bippes. Instability and transition of a three-dimensional boundary layer on a swept flat plate. Phys Fluids, 31:786-795, 1988. doi: 10.1063/1. 866814.

V. Ondrus, R. J. Meier, C. Klein, U. Henne, M. Schäferling, and U. Beifuß. Europium 1,3-di(thienyl)propane1,3-diones with outstanding properties for temperature sensing. Sensors and Actuators, A: Physical, 233:434-441, 2015. doi: 10.1016/j.sna.2015.07.023.

R. Petzold and R. Radespiel. Transition on a wing with spanwise varying crossflow and linear stability analysis. AIAA Journal, 53(2):321-335, 2015. doi: 10.2514/1. J053127.

B. Plogmann, W. Würz, and E. Krämer. Transitional flow in the wake of a moderate to large height cylindrical roughness element. Experiments in Fluids, 56:212, 2015. doi: $10.1007 / \mathrm{s} 00348-015-2069-5$.

A. Quast. Detection of transition by infrared image technique. In ICIASF '87 Record, pages 125-134. IEEE Publication, 1987.

R.H. Radetzsky, M.S. Reibert, and W.S. Saric. Effect of isolated micron-sized roughness on transition in sweptwing flows. AIAA Journal, 37:1370-1377, 1999. doi: $10.2514 / 2.635$.

G. Redecker and G. Wichmann. Forward sweep - a favourable concept for laminar flow. Journal of Aircraft, 28:97-103, 1991. doi: 10.2514/3.45997.

W.S. Saric, H.L. Reed, and E.B. White. Stability and transition of three-dimensional boundary layers. Annual Review of Fluid Mechanics, 35:413-440, 2003. doi: 10.1146/ annurev.fluid.35.101101.161045.

W.S. Saric, A.L. Carpenter, and H.L. Reed. Passive control of transition in three-dimensional boundary layers, with emphasis on discrete roughness elements. Phil. Trans. $R$. Soc. A, 369:1352-1364, 2011. doi: 10.1098/rsta.2010.0368.

W.S. Saric, D.E. West, M.W. Tufts, and H.L. Reed. Flight test experiments on discrete roughness element technology for laminar flow control. In 53rd AIAA Aerospace Sciences Meeting, AIAA SciTech Forum. AIAA 2015-0539, 2015. doi: 10.2514/6.2015-0539.

G. Schrauf. Status and perspectives of laminar flow. The Aeronautical Journal, 109:639-644, 2005. doi: 10.1017/ S000192400000097X.

G. Schrauf and L.O. Furtado Ferreira. Investigation for feasibility of a roughness test in the wind tunnel of the University of Stuttgart. Airbus Communication, 2011.

G. Schrauf, J. Perraud, D. Vitiello, and F. Lam. Comparison of boundary-layer transition predictions using flight test data. Journal of Aircraft, 35:891-897, 1998. doi: 10.2514/2. 2409. 
T. Streit and C. Hoffrogge. DLR transonic inverse design code extensions and modifications to increase versatility and robustness. The Aeronautical Journal, 121:1733-1757, 2017. doi: $10.1017 /$ aer.2017.101.

P. Wassermann and M.J. Kloker. Mechanisms and passive control of crossflow-vortex-induced transition in a threedimensional boundary layer. Journal of Fluid Mechanics, 456:49-84, 2002. doi: 10.1017/S0022112001007418.

A. Weiss, A.D. Gardner, C. Klein, and M. Raffel. Boundarylayer transition measurements on Mach-scaled helicopter rotor blade in climb. CEAS Aeronaut J, 8:613-623, 2017.

F.M. White and I. Corfield. Viscous fluid flow. McGraw-Hill, New York, 2nd edition, 1991.

M. Woodruff, W.S. Saric, and H.L. Reed. Receptivity measurements on a swept-wing model. In 41st AIAA Fluid Dynamics Conference and Exhibit, 27 - 30 June 2011, Honolulu, Hawaii. AIAA 2011-3882, 2011. doi: 10.2514/6. 2011-3882.

F.X. Wortmann and D. Althaus. Der Laminarwindkanal des Instituts für Aero- und Gasdynamik an der Technischen Hochschule Stuttgart. Zeitschriften für Flugwissenschaften, 12:225-248, 1964. 